\title{
Synthesis and Properties of Multi-Block Copolymers Based on Poly(oxyethylene)s and Aromatic Polyamides
}

\author{
Yoshio ImaI, Mikio KaJiYama, Shin-ichi Ogata, \\ and Masa-aki KAKIMOTO \\ Department of Textile and Polymeric Materials, \\ Tokyo Institute of Technology, Meguro-ku, Tokyo 152, Japan
}

(Received January 17, 1984)

\begin{abstract}
Polyether-aramid multi-block copolymers having high structural regularity were synthesized by the direct polycondensation of poly(oxyethylene)dicarboxylic acids with amineterminated telechelic aromatic polyamide (aramid) oligomers using triphenyl phosphite and pyridine as the condensing agent. The polymerizations at a total oligomer concentration of $100-$ $200 \mathrm{gl}^{-1}$ in a mixture of pyridine and $N$-methyl-2-pyrrolidone afforded the block copolymers having inherent viscosities of $0.4-0.9 \mathrm{dl} \mathrm{g}^{-1}$. These multi-block copolymers are soluble in $N, N$ dimethylacetamide (DMAc) and gave transparent, ductile, and elastomeric films by casting from DMAc solutions. Moisture regain of these films is $5-6 \%$ in $60 \%$ relative humidity at $25^{\circ} \mathrm{C}$. On the DTA curves in nitrogen, these copolymers exhibited two strong exothermic peaks at around 270 and $410^{\circ} \mathrm{C}$; the former temperature corresponds well to the initial weight loss temperature on the TG curves. In the polyether-aramid multi-block copolymers, tensile strength and modulus of the films fall with an increase in the poly(oxyethylene) content, whereas elongation at break increases.

KEY WORDS Polyether-Aramid Multi-Block Copolymers / Direct

Polycondensation / Solubility / Thermal Behavior / Tensile Properties /
\end{abstract}

Poly(oxyethylene)dicarboxylic acid (POEdiacid) is a new, commercially available, telechelic polymer with carboxyl groups at both terminal positions. Kimura et al. ${ }^{1}$ utilized the oligomeric diacids to synthesize polyetheramides by the melt polycondensation with aliphatic diamines. Recently we reported the synthesis of another type of polyether-amides with high molecular weights by the direct polycondensation of POE-diacids with aromatic diamines using triphenyl phosphite and pyridine as the condensing agent. ${ }^{2}$ As an ex- tension of the previous study, ${ }^{2}$ we have successfully prepared polyether-aramid multiblock copolymers of high molecular weights from combinations of POE-diacids and amineterminated telechelic aromatic polyamide (aramid) oligomers. Various types of polyetheramide block copolymers have been prepared, but the block copolymers having high structural regularity have not been described so far. $^{3,4}$ In this article, synthesis and characterization of the multi-block copolymers with well-defined structures are reported.

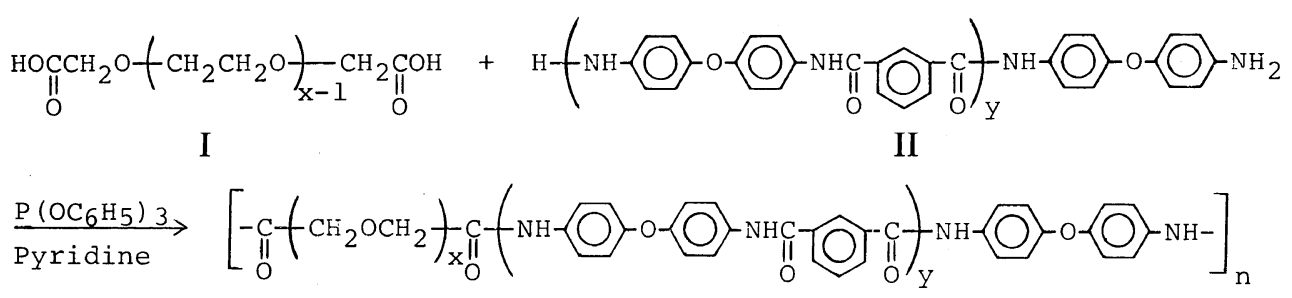




\section{EXPERIMENTAL}

\section{Materials}

POE-diacids were supplied by Kawaken Fine Chemicals Corp. Bis(4-aminophenyl) ether (ODA) and isophthalic acid (IPA) were purified by recrystallization from tetrahydrofuran and water, respectively. Triphenyl phosphite, pyridine, $\quad N$-methyl-2-pyrrolidone (NMP), and $N, N$-dimethylacetamide (DMAc) were purified by distillation. Other inorganic reagents were used as received.

\section{Synthesis of Amine-Terminated Telechelic Aramid Oligomers}

Aramid Oligomer IIa. A mixture of $10.00 \mathrm{~g}$ $(0.05 \mathrm{~mol})$ of ODA, $6.20 \mathrm{~g}(0.0375 \mathrm{~mol})$ of IPA, $27 \mathrm{ml}$ of triphenyl phosphite, $5 \mathrm{~g}$ of lithium chloride, and $2.5 \mathrm{~g}$ of calcium chloride in $50 \mathrm{ml}$ of pyridine and $100 \mathrm{ml}$ of NMP was heated with stirring at $100^{\circ} \mathrm{C}$ for $3 \mathrm{~h}$ under nitrogen. The reaction mixture was poured into 41 of methanol, and the precipitate was collected. The product was purified by three reprecipitations from the DMAc-methanol system, followed by thorough washing with hot ethanol, and dried at $30^{\circ} \mathrm{C}$ in vacuo. The yield was $12.9 \mathrm{~g}(87 \%)$. Inherent viscosity of the aramid oligomer in DMAc was $0.11 \mathrm{dlg}^{-1}$ at $30^{\circ} \mathrm{C}$. The infrared (IR) spectrum (KBr) exhibited absorptions at $3270 \mathrm{~cm}^{-1}(\mathrm{~N}-\mathrm{H}), 1645 \mathrm{~cm}^{-1}$ $(\mathrm{C}=\mathrm{O})$, and $1230 \mathrm{~cm}^{-1}(\mathrm{C}-\mathrm{O}-\mathrm{C})$.

Other aramid oligomers IIb and IIc were prepared by analogous procedures.

\section{Synthesis of Multi-Block Copolymers}

Block Copolymer E75A5. A mixture of $1.36 \mathrm{~g}(0.4 \mathrm{mmol})$ of $\mathrm{Ib}, 0.80 \mathrm{~g}(0.4 \mathrm{mmol})$ of IIa, $0.3 \mathrm{ml}(1.1 \mathrm{mmol})$ of triphenyl phosphite, $0.5 \mathrm{~g}$ of lithium chloride, and $0.25 \mathrm{~g}$ of calcium chloride in $5 \mathrm{ml}$ of pyridine and $10 \mathrm{ml}$ of NMP was stirred at $100^{\circ} \mathrm{C}$ for $3 \mathrm{~h}$ under nitrogen. The resultant polymer was isolated by pouring the reaction solution into $500 \mathrm{ml}$ of methanol. The product was purified as described for aramid oligomers. The polymer weighed $1.84 \mathrm{~g}$
$(86 \%)$ and had an inherent viscosity of 0.64 $\mathrm{dlg}^{-1}$ in DMAc. The IR spectrum (film) showed absorptions at $3300 \mathrm{~cm}^{-1}(\mathrm{~N}-\mathrm{H})$, $1660 \mathrm{~cm}^{-1}(\mathrm{C}=\mathrm{O})$, and 1225 and $1100 \mathrm{~cm}^{-1}$ (C-O-C).

All other block copolymers were synthesized by the same procedure.

\section{Measurements}

IR spectra were recorded on a Hitachi EPG3 spectrophotometer. Differential thermal analysis (DTA) and thermogravimetry (TG) were performed with Shimadzu thermal analyzers DTA-30M and TGA-30M, respectively. Tensile properties such as strength and elongation were determined from stress-strain curves obtained with a Toyo Baldwin Tensilon UTM-III at an elongation rate of $20 \% \mathrm{~min}^{-1}$. Measurements were performed at room temperature with film specimens $(1.0 \mathrm{~cm}$ wide, $5.0 \mathrm{~cm}$ long, and about $0.1 \mathrm{~mm}$ thick) and average of at least five individual determinations was taken.

\section{RESULTS AND DISCUSSION}

\section{Synthesis of Amine-Terminated Telechelic} Aramid Oligomers

To obtain condensation block copolymers having high structural regularity, it is necessary to synthesize both block species with terminal functional groups prior to polycondensation. In the present study, amineterminated telechelic aramid block species were prepared and subsequently condensed with an equimolar amount of commercially available, carboxyl-terminated telechelic polyether blocks, POE-diacids, to give polyetheraramid multi-block copolymers.

Table I shows the molecular weight $\left(M_{n}\right)$ determined by titration, the degree of polymerization $(\bar{x})$, and the inherent viscosity of POE-diacids Ia - c employed.

For the aramid components, a combination of ODA and IPA was selected to obtain aramid oligomers having high solubility in 
Table I. Characterization of POE-diacids

\begin{tabular}{ccrc}
\hline & & & $\eta_{\text {inh }}{ }^{\mathrm{b}}$ \\
\cline { 3 - 4 } POE-diacid & $M_{n}{ }^{\mathrm{a}}$ & $\bar{x}$ & $\mathrm{dlg}^{-1}$ \\
\hline Ia & 1000 & 20 & 0.05 \\
Ib & 3400 & 75 & 0.10 \\
Ic & 7600 & 170 & 0.17 \\
\hline
\end{tabular}

a Determined by titration.

b Measured at a concentration of $0.5 \mathrm{~g} \mathrm{dl}^{-1}$ in DMAc at $30^{\circ} \mathrm{C}$.

amide-type solvents. Amine-terminated telechelic aramid blocks IIa - c were prepared by the direct condensation using triphenyl phosphite and pyridine as the condensing agent (eq 2).
In general, high-polymer formation is favored by an equimolar balance of the coreactants; an excess of either of the coreactants results in chain termination. In view of the fact that the aramid block species used in this work are of relatively high molecular weights, end group analysis by titration and elemental analysis may not be sufficiently accurate to determine the optimum ratio of the coreactant species. A series of experiments was then designed for this purpose. The results of the polycondensation between POE-diacid Ib and amine-terminated aramid oligomer $\mathrm{IIa}-\mathrm{c}$ conducted with a number of different block ratios are shown in Figure 1, where the extreme sensitivity of the copolymer inherent viscosity to the ratio of the coreacting blocks is

$$
\begin{aligned}
& \longrightarrow \\
& \longrightarrow
\end{aligned}
$$

II

apparent. At peaks of the composition-inherent viscosity curves, there must be equivalent numbers of end groups present in the two oligomers. ${ }^{5}$ Therefore,

$$
M_{n}(\text { aramid })=M_{n}(\mathrm{POE}) \times \frac{W(\text { aramid })}{W(\mathrm{POE})}
$$

where $W$ and $M_{n}$ are the weight and number average molecular weight, respectively, of the two blocks. From both Figure 1 and eq 3 with substituting 3400 for $M_{n}$ (POE), the molecular weights of amine-terminated aramid oligomers IIa, IIb, and IIc are estimated to be 2000, 3300, and 5400 , respectively. Table II shows the molecular weight $\left(M_{n}\right)$, the degree of polymerization $(\bar{y})$, the inherent viscosity, and the elemental analysis of the aramid oligomers.

\section{Synthesis of Polyether-Aramid Multi-Block Copolymers}

The direct polycondensation of POE-diacids

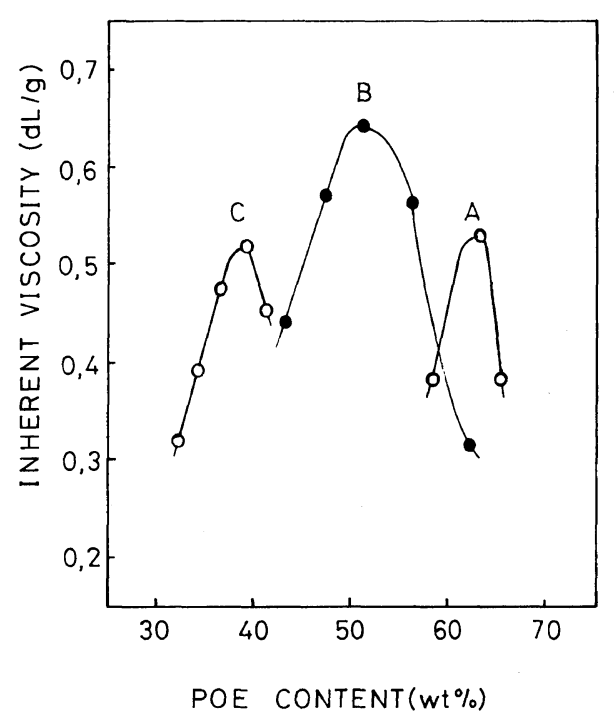

Figure 1. Influence of block ratio on inherent viscosity of the polyether-aramid multi-block copolymers formed in the polycondensation of POE-diacid Ib with aramid oligomer IIa (A), IIb (B), and IIc (C). 
Table II. Characterization of amine-terminated telechelic aramid oligomers

\begin{tabular}{|c|c|c|c|c|c|c|c|c|c|}
\hline \multirow{3}{*}{$\begin{array}{l}\text { Aramid } \\
\text { oligomer }\end{array}$} & \multirow{3}{*}{$M_{n}^{\mathrm{a}}$} & \multirow{3}{*}{$\bar{y}$} & \multirow{3}{*}{$\frac{\eta_{\mathrm{inh}}^{\mathrm{b}}}{\mathrm{dl} \mathrm{g}^{-1}}$} & \multicolumn{6}{|c|}{ Elemental analysis } \\
\hline & & & & \multicolumn{3}{|c|}{ Calcd $/ \%$} & \multicolumn{3}{|c|}{ Found $/ \%$} \\
\hline & & & & $\mathrm{C}$ & $\mathrm{H}$ & $\mathrm{N}$ & $\mathrm{C}$ & $\mathrm{H}$ & $\mathbf{N}$ \\
\hline IIa & 2000 & 5 & 0.11 & 72.61 & 4.54 & 9.41 & 72.49 & 4.16 & 8.98 \\
\hline IIb & 3300 & 9 & 0.17 & 72.66 & 4.40 & 8.99 & 72.47 & 4.33 & 8.68 \\
\hline IIc & 5400 & 16 & 0.24 & 72.69 & 4.33 & 8.75 & 72.43 & 4.22 & 8.33 \\
\hline
\end{tabular}

a Calculated from eq 3.

b Measured at a concentration of $0.5 \mathrm{~g} \mathrm{dl}^{-1}$. in DMAc at $30^{\circ} \mathrm{C}$.

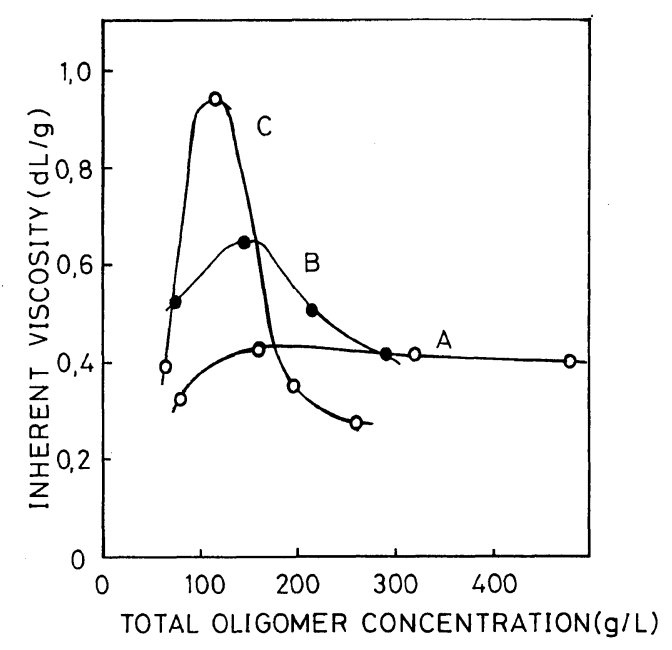

Figure 2. Influence of total oligomer concentration on inherent viscosity of polyether-aramid multi-block copolymers formed in the polycondensation of aramid oligomer IIa with POE-diacid Ia (A), Ib (B), and Ic (C).

Ia $-c$ with amine-terminated aramide oligomers IIa $-c$ giving polyether-aramid multiblock copolymers III was carried out in NMP using triphenyl phosphite and pyridine as the condensing agent (eq 1). The resultant multiblock copolymers are coded as ExAy, where the symbols $\mathrm{x}$ and $\mathrm{y}$ denote, respectively, the average degree of polymerization of a POEdiacid and that of an aramid oligomer.

To obtain the block copolymers with high molecular weights, the effects of oligomer concentration in the reaction mixture was investigated in detail. As shown in Figure 2, the total oligomer concentration, that is, the total
Table III. Synthesis of polyether-aramid multi-block copolymers

\begin{tabular}{|c|c|c|c|c|}
\hline \multicolumn{2}{|c|}{ Oligomers } & \multirow{3}{*}{$\frac{\begin{array}{c}\text { POE } \\
\text { content }^{\mathrm{a}}\end{array}}{\mathrm{wt} \%}$} & \multicolumn{2}{|c|}{ Block copolymer } \\
\hline \multirow{2}{*}{$\begin{array}{l}\text { POE- } \\
\text { diacid }\end{array}$} & \multirow{2}{*}{ Aramid } & & \multirow{2}{*}{ Code } & \multirow{2}{*}{$\frac{\eta_{\mathrm{inh}}^{\mathrm{b}}}{\mathrm{d} l \mathrm{~g}^{-1}}$} \\
\hline & & & & \\
\hline Ia & IIa & 33 & E20A5 & 0.42 \\
\hline Ia & $\mathrm{IIb}$ & 23 & E20A6 & 0.44 \\
\hline Ia & IIc & 16 & E20A16 & 0.50 \\
\hline $\mathrm{Ib}$ & IIa & 63 & E75A5 & 0.64 \\
\hline $\mathrm{Ib}$ & IIb & 51 & E75A9 & 0.65 \\
\hline Ib & IIc & 39 & E75A16 & 0.52 \\
\hline Ic & IIa & 79 & E170A5 & 0.93 \\
\hline Ic & IIb & 70 & E170A9 & 0.90 \\
\hline Ic & IIc & 58 & E170A16 & 0.81 \\
\hline
\end{tabular}

a $W(\mathrm{POE}) /[W(\mathrm{POE})+W($ aramid $)]$ in feed.

b Measured at a concentration of $0.5 \mathrm{~g} \mathrm{dl}^{-1}$ in DMAc at $30^{\circ} \mathrm{C}$.

amount of the POE-diacid and the aramid oligomer in a mixture of pyridine and NMP, greatly influenced the inherent viscosity of the polymers. For all the polymerizations, 100 $200 \mathrm{gl}^{-1}$ oligomer concentration was found to be optimum and the inherent viscosity values reached as high as $0.9 \mathrm{dl} \mathrm{g}^{-1}$.

Under these polymerization conditions, various types of multi-block copolymers with inherent viscosities of $0.4-0.9 \mathrm{dlg}^{-1}$ were obtained readily, suggesting that the block copolymers are sufficiently high molecular weight materials (Table III).

The structure of the resulting polymers was confirmed by IR spectroscopy and elemental 
Table IV. Elemental analysis of polyether-aramid multi-block copolymers

\begin{tabular}{lllllllll}
\hline \multirow{2}{*}{$\begin{array}{c}\text { Block } \\
\text { copolymer }\end{array}$} & \multicolumn{3}{c}{ Calcd/\% } & & \multicolumn{3}{c}{ Found/\% } \\
\cline { 2 - 5 } \cline { 7 - 9 } & $\mathrm{C}$ & $\mathrm{H}$ & $\mathrm{N}$ & & $\mathrm{C}$ & $\mathrm{H}$ & $\mathrm{N}$ \\
\hline E20A9 & 68.35 & 5.29 & 6.77 & & 67.19 & 5.36 & 6.38 \\
E75A5 & 61.16 & 7.23 & 3.35 & & 59.36 & 7.04 & 2.79 \\
E75A9 & 63.40 & 6.65 & 4.35 & & 61.76 & 6.57 & 3.88 \\
E75A16 & 65.63 & 6.08 & 5.33 & & 64.17 & 5.85 & 4.80 \\
E170A5 & 58.27 & 8.04 & 1.88 & & 59.51 & 7.71 & 2.35 \\
\hline
\end{tabular}

analysis (Table IV). In the IR spectra, characteristic amide absorptions at $3300 \mathrm{~cm}^{-1}(\mathrm{~N}-\mathrm{H})$ and $1660 \mathrm{~cm}^{-1}(\mathrm{C}=\mathrm{O})$, aromatic absorptions at 1600 and $1490 \mathrm{~cm}^{-1}$ due to aramid linkages, and two ether absorptions at 1230 and 1100 $\mathrm{cm}^{-1}$ appeared, whereas a strong carbonyl absorption at $1740 \mathrm{~cm}^{-1}$ due to the carboxylic acid function of the starting POE-diacids disappeared. The IR spectra are almost identical with those of the polyether-amides reported previously. ${ }^{2}$

\section{Properties of Polyether-Aramid Multi-Block} Copolymers

The multi-block copolymers ExAy are readily soluble in DMAc, but insoluble in other organic solvents including NMP, pyridine, $m$ cresol, formic acid, tetrahydrofuran, acetone, methanol, chloroform, benzene, and water. This solubility behavior is fairly comparable to that of the aramid oligomers, in contrast to the good solubility nature of the POE-diacids in a wide range of solvents. Transparent, ductile, and elastomeric films could be cast from DMAc solutions.

Moisture regain of these block copolymers in the form of films in $60 \%$ relative humidity at $25^{\circ} \mathrm{C}$ was determined to be in the range of 5 $6 \%$. These values are compared with those of 8.5 and $1.4 \%$ for the parent POE homopolymer $\left(M_{n}, 20000\right)$ and poly $\left(4,4^{\prime}\right.$-oxydi- $p$ phenylene isophthalamide) (aramid homopolymer IId, $\eta_{\text {inh }}: 2.01 \mathrm{dlg}^{-1}$ in DMAc), respectively.

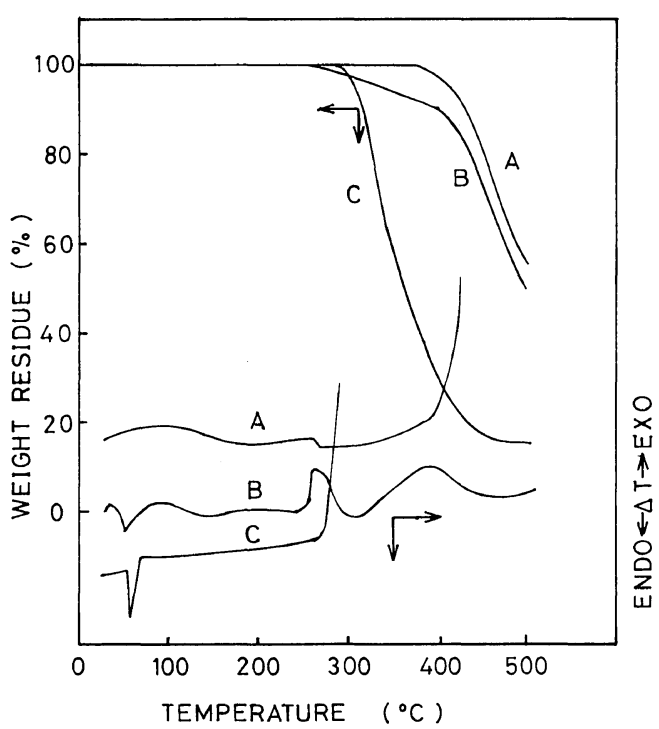

Figure 3. DTA and TG curves in nitrogen for (A) aramid homopolymer, (B) multi-block copolymer E170A16, and (C) POE homopolymer: DTA heating rate, $20^{\circ} \mathrm{C} \min ^{-1}$; TG heating rate, $5^{\circ} \mathrm{C} \min ^{-1}$.

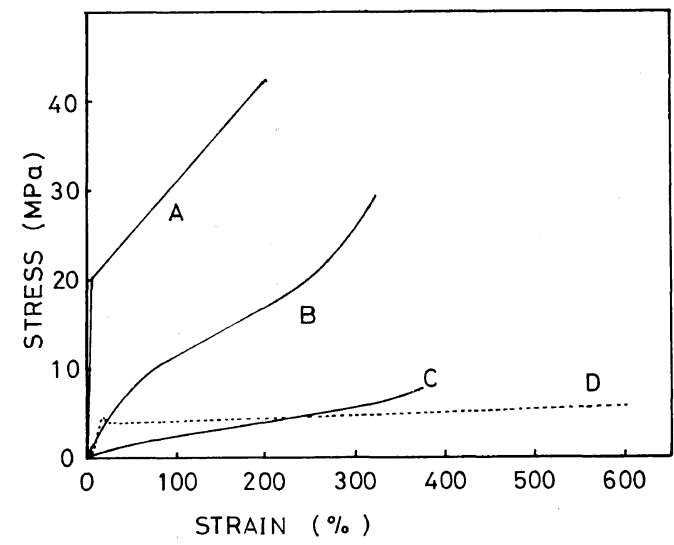

Figure 4. Stress-strain curves for polyether-aramid multi-block copolymer E75A16 (A), E75A9 (B), E75A5 (C), and E170A5 (D).

The thermal behavior of the polyetheraramid multi-block copolymers was evaluated by DTA and TG. Typical DTA and TG curves for the representative multi-block copolymer, E170A16, as well as for the parent POE homopolymer and aramid homopolymer IId, are shown in Figure 3.

On the DTA curves in nitrogen, all the 
Table V. Tensile properties of cast films of polyether-aramid multi-block copolymers

\begin{tabular}{|c|c|c|c|c|c|}
\hline \multicolumn{2}{|c|}{ Block copolymer } & \multirow{2}{*}{$\begin{array}{l}\text { Tnesile } \\
\text { strength }\end{array}$} & \multirow{2}{*}{$\begin{array}{l}\text { Elongation } \\
\text { at break }\end{array}$} & \multirow{2}{*}{$\begin{array}{l}\text { Tensile } \\
\text { modulus }\end{array}$} & \multirow{2}{*}{$\begin{array}{l}\text { Elastic } \\
\text { recovery }\end{array}$} \\
\hline & POE & & & & \\
\hline \multirow[t]{2}{*}{ Code } & & $\mathrm{MPa}$ & $\%$ & $\mathrm{MPa}$ & $\%$ \\
\hline & $\%$ & & & & \\
\hline E75A16 & 39 & 42 & 200 & 500 & 58 \\
\hline E75A9 & 51 & 29 & 320 & 470 & 86 \\
\hline E75A5 & 63 & 6.9 & 370 & 6 & 100 \\
\hline E170A9 & 70 & 7.1 & 580 & 42 & 53 \\
\hline E170A5 & 79 & 5.6 & 600 & 28 & 59 \\
\hline Aramid IId & - & 68 & 4 & 1700 & - \\
\hline
\end{tabular}

a Measured by $100 \%$ extension of film specimens and relaxation.

multi-block copolymers exhibited two strong exothermic peaks at around 270 and $410^{\circ} \mathrm{C}$ due to the decomposition of oxyethylene units and aramid linkages, respectively. In addition, a characteristic endothermic peak, probably due to the melting of POE crystallites, was observed in the range of $51-56^{\circ} \mathrm{C}$ for the E170Ay series block copolymers having increased POE block length. These melting temperatures are in good agreement with that of the POE homopolymer $\left(59^{\circ} \mathrm{C}\right)$. Aramid homopolymer IId showed glass transition temperature at $270^{\circ} \mathrm{C}$, but similar transition in these block copolymers could not be detected because of the appearance of a strong exothermic peak due to decomposition of the POE blocks in the same region. The TG curves of the multi-block copolymers were fairly similar in shape to those of the aramid homopolymer, though the block copolymers began to lose weight above $270^{\circ} \mathrm{C}$ in nitrogen.

Stress-strain curves of the polyether-aramid multi-block copolymer films are shown in Figure 4 and the tensile properties are summarized in Table V. Since these block copolymers are ductile and elastomeric, their tensile properties differ significantly from those of aramid homopolymer IId. The rigid structure of the aramid blocks is apparently retained in the block copolymers to produce strong elastomeric pseudo-crosslinked materials. A typical example is a snappy elastomer of E75A5 which displays a tensile strength of $6.9 \mathrm{MPa}$ with $370 \%$ elongation and $100 \%$ elastic recovery. In the E75Ay block copolymer series, tensile strength and modulus fall steeply with increasing POE concentrations, whereas elongation at break increases rather gradually. Similar structure-property effects have been observed with other block copolymer systems based on POE and polycarbonate. ${ }^{6-8}$

Morphology and other behavior of these polyether-aramid multi-block copolymers will be published in the near future.

\section{REFERENCES}

1. Y. Kimura, N. Sugihara, and I. Taniguchi, Macromolecules, 16, 1023 (1983).

2. Y. Imai, S. Ogata, and M. Kakimoto, Makromol. Chem., Rapid Commun., 5, 47 (1984).

3. R. J. Zdrahala, E. M. Firer, and J. F. Fellers, J. Polym. Sci., Polym. Chem. Ed., 15, 689 (1977).

4. L. Castaldo, G. Maglio, and R. Palumbo, J. Polym. Sci., Polym. Lett. Ed., 16, 643 (1978).

5. K. Riches and R. N. Haward, Polymer, 9, 103 (1968).

6. E. P. Goldberg, J. Polym. Sci., C, 4, 707 (1964).

7. S. H. Merril and S. E. Petrie, J. Polym. Sci., A, 3, 2189 (1965).

8. T. Suzuki and T. Kotaka, Macromolecules, 13, 1495 (1980). 